\title{
On Submanifolds of $N(k)$-Quasi Einstein Manifolds with a Type of Semi-Symmetric Metric Connection
}

\author{
İnan Ünal ${ }^{1}$ \\ ${ }^{1}$ Department of Computer Engineering, Faculty of Engineering, Munzur University, Tunceli, Turkey
}

\section{Article Info}

Keywords: $N(k)$-quasi Einstein manifolds, Totally geodesic, Totally umbilical, Para-Kenmotsu 2010 AMS: 53C15, 53C25, 53D10

Received: 24 September 2020

Accepted: 2 November 2020

Available online: 23 December 2020

\begin{abstract}
In this study, we consider the $N(k)$-quasi Einstein manifolds with respect to a type of semi-symmetric metric connection. We suppose that the generator of $N(k)$-quasi-Einstein manifolds is parallel with respect to semi-symmetric metric connection and we classify such manifolds. In addition, we consider the submanifolds of a $N(k)$-quasi Einstein manifold and we obtain some conditions on the totally geodesic and the totally umbilic submanifolds. Finally, we consider a para-Kenmotsu space form as an example of $N(k)$-quasi-Einstein manifolds.
\end{abstract}

\section{Introduction}

An Einstein manifold is a Riemannian manifold $(M, g)$ satisfying Einstein fields equation. We determine such manifold by $R i c=\lambda g$, for the Ricci curvature Ric of $M$ non-zero constant $\lambda$. In differential geometry, there are many kind of manifolds which satisfy this relation. Einstein manifolds are widely studied by researchers from mathematics and physics. A well known generalization of Einstein manifolds is the notion of quasi-Einstein manifolds defined by Chaki in [5]. Similar to Einstein manifolds, quasi-Einstein manifolds are also occur in the solutions of Einstein field equations. In this manner, quasi-Einstein manifolds have some applications in the general relativity. An example is Robertson-Walker space times [8]. A quasi-Einstein manifold is a Riemannian manifold $(M, g)$ which has the following relation on the Ricci tensor of $M$;

$$
\operatorname{Ric}\left(\Omega_{1}, \Omega_{2}\right)=a g\left(\Omega_{1}, \Omega_{2}\right)+b \eta\left(\Omega_{1}\right) \eta\left(\Omega_{2}\right)
$$

for some smooth functions $a$ and $b$, arbitrary vector fields $\Omega_{1}, \Omega_{2} \in \Gamma(T M)$, where $\eta$ is a non-zero $1-$ form on $M$ such that $g\left(\Omega_{1}, \xi\right)=$ $\eta\left(\Omega_{1}\right), \eta(\xi)=1$ for a vector field $\xi \in \Gamma(T M)$. We call $\eta$ by associated $1-$ form and $\xi$ by the generator of the manifold. If a ( $\left.2 m+1\right)$ dimensional Riemannian manifold $M$ has an almost contact metric structure $(\phi, \xi, \eta, g)$ and Ricci tensor satisfies $(1.1)$ then $M$ is called by an $\eta$-Einstein manifold [1]. So, an $\eta$-Einstein manifold is an example of quasi-Einstein manifolds. Also, a generalized Sasakian space form is a quasi-Einstein manifold [6].

$k$-nullity distribution of a quasi Einstein manifold is defined as

$$
N(k): p \longrightarrow N_{p}(k)=\left[\Omega_{3} \in \Gamma\left(T_{p} M\right): \operatorname{Rim}\left(\Omega_{1}, \Omega_{2}\right) \Omega_{3}=k\left\{g\left(\Omega_{2}, \Omega_{3}\right) \Omega_{1}-g\left(\Omega_{1}, \Omega_{3}\right) \Omega_{2}\right\}\right],
$$

for any $\Omega_{1}, \Omega_{2} \in \Gamma\left(T_{p} M\right)$ and $k \in \mathbb{R}$, where Rim is the Riemannian curvature tensor of $M$. If the generator vector field $\xi$ belongs to $k-$ nullity distribution then $M$ is called $N(k)$-quasi Einstein manifold $\left(N K(Q E)_{m}\right)$ [5]. A quasi Einstein manifold is an $N K(Q E)_{m}$ manifold if it is conformally flat [15]. In 2004 De and Ghosh [7] prove the existence of $N K(Q E)_{m}$ manifolds and presented some results. In 2008 Özgür [3] examined $N K(Q E)_{m}$ manifolds under some certain curvature conditions. Yıldız et al. [4] worked on $N K(Q E)_{m}$ manifolds with some semi-symmetry conditions and gave examples. The Riemannian geometry of $N(k)$ - quasi-Einstein manifolds have been studied by many researchers in $[3,6,10,12,16]$.

In this work, we consider a $N K(Q E)_{m}$ manifold admitting a type of semi-symmetric metric connection (SSMC) and we obtain some results on the submanifolds of such manifolds. Also, we present a classification of $N K(Q E)_{m}$ manifold admitting SSMC. We proved some theorems on the totally geodesic and totally umbilical submanifolds. Finally, we consider a para-Kenmotsu space form as an example. 


\section{N(k)-quasi Einstein manifolds with a type of semi-symmetric metric connection}

In the Riemannian geometry, we know that the Levi-Civita connection have no torsion and it is a metric connection. Also, there are many type of connections which has torsion and not symmetric. One of them is a semi-symmetric metric connection (SSMC) . In the [17] Yano defined a type of SSMC. Murathan and Özgür [3] studied Riemannian manifolds with this connection under some semi-symmetry conditions. The authors consider the parallel unit vector field with respect to the Levi-Civita connection. In this section, we consider a $N K(Q E)_{m}$ manifold with the parallel vector field $\xi$ with respect to SSMC. We present some results related to SSMC.

Let $M$ be an $m$-dimensional $N K(Q E)_{m}$ manifold and define a map on $M$ by

$$
\overline{\widetilde{\nabla}}_{\Omega_{1}} \Omega_{2}=\widetilde{\nabla}_{\Omega_{1}} \Omega_{2}+\eta\left(\Omega_{2}\right) \Omega_{1}-g\left(\Omega_{1}, \Omega_{2}\right) \xi
$$

for all $\Omega_{1}, \Omega_{2} \in \Gamma(T M)$, where $\widetilde{\nabla}$ is the Levi-Civita connection (LCC) on $M$. The map $\bar{\nabla}$ on $M$ defines a semi-symmetric metric connection [17]. The Riemannian curvature of $M$ with respect to $\overline{\widetilde{\nabla}}$ was obtained in [17] as;

$$
\begin{aligned}
\widetilde{\operatorname{Rim}}\left(\Omega_{1}, \Omega_{2}, \Omega_{3}, \Omega_{4}\right) & =\widetilde{\operatorname{Rim}}\left(\Omega_{1}, \Omega_{2}, \Omega_{3}, \Omega_{4}\right)-\omega\left(\Omega_{2}, \Omega_{3}\right) g\left(\Omega_{1}, \Omega_{4}\right)+\omega\left(\Omega_{1}, \Omega_{3}\right) g\left(\Omega_{2}, \Omega_{4}\right) \\
& -g\left(\Omega_{2}, \Omega_{3}\right) \omega\left(\Omega_{1}, \Omega_{4}\right)+g\left(\Omega_{1}, \Omega_{3}\right) \omega\left(\Omega_{2}, \Omega_{4}\right)
\end{aligned}
$$

for all $\Omega_{1}, \Omega_{2}, \Omega_{3}, \Omega_{4} \in \Gamma(T M)$, where $\omega$ is defined as

$$
\omega\left(\Omega_{1}, \Omega_{2}\right)=\left(\widetilde{\nabla}_{\Omega_{1}} \eta\right) \Omega_{2}-\eta\left(\Omega_{1}\right) \eta\left(\Omega_{2}\right)+\frac{1}{2} g\left(\Omega_{1}, \Omega_{2}\right) .
$$

From (2.1) we obtain

$$
\overline{\widetilde{\nabla}}_{\Omega_{1}} \xi=\widetilde{\nabla}_{\Omega_{1}} \xi+\Omega_{1}-\eta\left(\Omega_{1}\right) \xi .
$$

Suppose that $\bar{\nabla}_{\Omega_{1}} \xi=0$. Then, we recall $\xi$ by parallel vector field with respect to SSMC. Thus, we get

$$
\widetilde{\nabla}_{\Omega_{1}} \xi=-\Omega_{1}+\eta\left(\Omega_{1}\right) \xi
$$

On the other hand, we have

$$
\left(\widetilde{\nabla}_{\Omega_{1}} \eta\right) \Omega_{2}=\widetilde{\nabla}_{\Omega_{1}} \eta\left(\Omega_{2}\right)-\eta\left(\widetilde{\nabla}_{\Omega_{1}} \Omega_{2}\right) .
$$

Since, $\widetilde{\nabla}$ is a metric connection i.e $\left(\widetilde{\nabla}_{\Omega_{1}} g\right)\left(\Omega_{2}, \Omega_{3}\right)=g\left(\widetilde{\nabla}_{\Omega_{1}} \Omega_{2}, \Omega_{3}\right)+g\left(\Omega_{3}, \widetilde{\nabla}_{\Omega_{1}} \Omega_{2}\right)$, from (2.3) we get

$$
\left(\tilde{\nabla}_{\Omega_{1}} \eta\right) \Omega_{2}=-g\left(\Omega_{1}, \Omega_{2}\right)+\eta\left(\Omega_{1}\right) \eta\left(\Omega_{2}\right) .
$$

Thus, we obtain $\omega\left(\Omega_{1}, \Omega_{2}\right)=-\frac{1}{2} g\left(\Omega_{1}, \Omega_{2}\right)$ and so from (2.2), we get

$$
\widetilde{\widetilde{R i m}}\left(\Omega_{1}, \Omega_{2}, \Omega_{3}, \Omega_{4}\right)=\widetilde{\operatorname{Rim}}\left(\Omega_{1}, \Omega_{2}, \Omega_{3}, \Omega_{4}\right)+g\left(\Omega_{2}, \Omega_{3}\right) g\left(\Omega_{1}, \Omega_{4}\right)-g\left(\Omega_{1}, \Omega_{3}\right) g\left(\Omega_{2}, \Omega_{4}\right) .
$$

In [2] it was proved that in a $N K(Q E)_{m}$ manifold $k=\frac{a+b}{m-1}$. Thus, from (1.2), we obtain

$$
\overline{\widetilde{R}}\left(\Omega_{1}, \Omega_{2}, \Omega_{3}, \Omega_{4}\right)=\left(\frac{a+b}{m-1}+1\right)\left[g\left(\Omega_{2}, \Omega_{3}\right) g\left(\Omega_{1}, \Omega_{4}\right)-g\left(\Omega_{1}, \Omega_{3}\right) g\left(\Omega_{2}, \Omega_{4}\right)\right]
$$

Finally, we state that

Theorem 2.1. Let $M$ be a $N K(Q E)_{m}$ manifold with respect to a SSMC $\overline{\widetilde{\nabla}}$ and $\xi$ be a parallel vector field with respect to $\overline{\widetilde{\nabla}}$. We have following classifications;

- If $a+b=1-m$ then $M$ is locally isometric to $m$-dimensional Euclidean space $\mathbb{E}^{m}$,

- If $a+b>1-m$ then $M$ is locally isometric to $m$-dimensional sphere $S^{m}\left(\frac{a+b}{m-1}+1\right)$,

- If $a+b<1-m$ then $M$ is locally isometric to $m$-dimensional hyperbolic space $H^{n}\left(\frac{a+b}{m-1}+1\right)$.

Let take an orthonormal basis of $M$ as $\left\{E_{1}, E_{2}, \ldots, E_{m-1}, E_{m}=\xi\right\}$. Then with taking sum over $1 \leq i \leq m$ in (2.4) we obtain

$$
\sum_{i=1}^{m} \widetilde{\widetilde{\operatorname{Rim}}}\left(\Omega_{1}, E_{i}, E_{i}, \Omega_{4}\right)=\sum_{i=1}^{m}\left\{\widetilde{\operatorname{Rim}}\left(\Omega_{1}, E_{i}, E_{i}, \Omega_{4}\right)+g\left(E_{i}, E_{i}\right) g\left(\Omega_{1}, \Omega_{4}\right)-g\left(\Omega_{1}, E_{i}\right) g\left(E_{i}, \Omega_{4}\right)\right\}
$$

and so, we get

$$
\widetilde{\widetilde{R i c}}\left(\Omega_{1}, \Omega_{4}\right)=\widetilde{\operatorname{Ric}}\left(\Omega_{1}, \Omega_{4}\right)+(m-1) g\left(\Omega_{1}, \Omega_{4}\right)
$$

for all $\Omega_{1}, \Omega_{2} \in \Gamma(T M)$. Then from (1.1), we obtain

$$
\widetilde{\widetilde{R i c}}\left(\Omega_{1}, \Omega_{4}\right)=(a+(m-1)) g\left(\Omega_{1}, \Omega_{4}\right)+b m \eta\left(\Omega_{1}\right) \eta\left(\Omega_{2}\right)
$$

Finally, we conclude that;

Theorem 2.2. Let $M$ be an $N K(Q E)_{m}$ manifold with respect to a LCC $\tilde{\nabla}$ and $\xi$ be a parallel vector field with respect to SSMC $\bar{\nabla}$. Then $M$ is an $N K(Q E)_{m}$ manifold with respect to $\widetilde{\widetilde{\nabla}}$. 


\section{Submanifolds of $\mathbf{N}(\mathbf{k})$-quasi Einstein manifolds with a type of semi-symmetric metric connection}

Let $M$ be an $m$-dimensional $N K(Q E)_{m}$ manifold with respect to SSMC $\overline{\widetilde{\nabla}}$ and $N$ be an $n$-dimensional submanifold of $M$. Suppose that the generator vector field $\xi$ tangent to $N$. Thus, we have two subbundles of $T M$ as $T N$ and $T N^{\perp}$ such that $T M=T N \oplus T N^{\perp}$. The subbundles $T N$ and $T N^{\perp}$ are called tangent bundle and normal bundle of $N$, respectively. Let recall some classical equations from the submanifold theory. For details we refer to reader [1].

The Gauss equation is given by

$$
\widetilde{\nabla}_{\Omega_{1}} \Omega_{2}=\nabla_{\Omega_{1}} \Omega_{2}+\sigma\left(\Omega_{1}, \Omega_{2}\right)
$$

for all $\Omega_{1}, \Omega_{2} \in \Gamma(T N)$, where $\sigma\left(\Omega_{1}, \Omega_{2}\right)$ denote the second fundamental form, and $\widetilde{\nabla}, \nabla$ are the Levi-Civita connections on $M$ and $N$, respectively.

The Weingarten equation is

$$
\widetilde{\nabla}_{\Omega_{1}} W=-A_{W} \Omega_{1}+\nabla_{\Omega_{1}}^{\perp} W
$$

for all $\Omega_{1} \in \Gamma(T N)$ and $W \in \Gamma\left(T N^{\perp}\right)$, where $A_{\underline{W}}$ is the shape operator related to $W, \nabla^{\perp}$ is the induced normal connection on the normal bundle $T N^{\perp}$. Consider the definition of SSMC $\widetilde{\widetilde{\nabla}}$ and using the Gauss equation, we get

$$
\overline{\widetilde{\nabla}}_{\Omega_{1}} \Omega_{2}=\nabla_{\Omega_{1}} \Omega_{2}+\eta\left(\Omega_{2}\right) \Omega_{1}-g\left(\Omega_{1}, \Omega_{2}\right) \xi+\sigma\left(\Omega_{1}, \Omega_{2}\right) .
$$

Suppose that $\xi$ is parallel with respect to $\bar{\nabla}$, then we obtain

$$
\nabla_{\Omega_{1}} \xi=-\Omega_{1}+\eta\left(\Omega_{1}\right) \xi-\sigma\left(\Omega_{1}, \xi\right) .
$$

Hence, we provide the following lemma.

Lemma 3.1. Let $M$ be an $N K(Q E)_{m}$ manifold with respect to $S S M C \tilde{\nabla}, N$ be a submanifold of $M$, and $\xi$ be a parallel vector field with respect to $S S M C \overline{\widetilde{\nabla}}$. Then, we get

$$
\nabla_{\Omega_{1}} \xi=-\Omega_{1}+\eta\left(\Omega_{1}\right) \xi, \sigma\left(\Omega_{1}, \xi\right)=0
$$

for all $\Omega_{1} \in \Gamma(T N)$, where $\xi \in \Gamma(T N)$.

Also, we know that

$$
\left(\widetilde{\nabla}_{\Omega_{1}} \sigma\right)\left(\Omega_{2}, \Omega_{3}\right)=\nabla_{\Omega_{1}}^{\perp}\left(\sigma\left(\Omega_{1}, \Omega_{2}\right)\right)-\sigma\left(\nabla_{\Omega_{1}} \Omega_{2}, \Omega_{3}\right)-\sigma\left(\Omega_{2}, \nabla_{\Omega_{1}} \Omega_{3}\right)
$$

for all $\Omega_{1}, \Omega_{2}, \Omega_{3} \in \Gamma(T N)[1]$.

Definition 3.2. Let $M$ be an $N K(Q E)_{m}$ manifold and $N$ be submanifold of $M$. If the covariant derivation of the second fundamental form vanishes, then $N$ is called parallel submanifold [1].

Theorem 3.3. Let $M$ be an $N K(Q E)_{m}$ manifold with respect to $S S M C \overline{\widetilde{\nabla}}, N$ be a submanifold of $M$ and $\xi$ be a parallel vector field with respect to SSMC $\overline{\widetilde{\nabla}}$. If N is parallel submanifold with respect to LCC $\widetilde{\nabla}$ then it is not parallel submanifold with respect to SSMC $\overline{\widetilde{\nabla}}$.

Proof. From the definition of SSMC $\overline{\widetilde{\nabla}}$, we have

$$
\begin{aligned}
\left(\widetilde{\nabla}_{\Omega_{1}} \sigma\right)\left(\Omega_{2}, \Omega_{3}\right) & =\widetilde{\nabla}_{\Omega_{1}} \sigma\left(\Omega_{1}, \Omega_{2}\right)-\sigma\left(\widetilde{\nabla}_{\Omega_{1}} \Omega_{2}, \Omega_{3}\right)-\eta\left(\Omega_{2}\right) \sigma\left(\Omega_{1}, \Omega_{3}\right)-g\left(\Omega_{1}, \Omega_{2}\right) \sigma(\xi, Z) \\
& -\sigma\left(\Omega_{2}, \tilde{\nabla}_{\Omega_{1}} \Omega_{3}\right)-\eta\left(\Omega_{3}\right) \sigma\left(\Omega_{1}, \Omega_{2}\right)-g\left(\Omega_{1}, \Omega_{3}\right) \sigma\left(\Omega_{2}, \xi\right) .
\end{aligned}
$$

Since $\xi$ is parallel with respect to SSMC $\overline{\widetilde{\nabla}}$, by using Lemma 3.1 we obtain

$$
\left(\overline{\widetilde{\nabla}}_{\Omega_{1}} \sigma\right)\left(\Omega_{2}, \Omega_{3}\right)=\nabla_{\Omega_{1}}^{\perp}\left(\sigma\left(\Omega_{1}, \Omega_{2}\right)\right)-\sigma\left(\nabla_{\Omega_{1}} \Omega_{2}, \Omega_{3}\right)-\sigma\left(\Omega_{2}, \nabla_{\Omega_{1}} \Omega_{3}\right)-\eta\left(\Omega_{2}\right) \sigma\left(\Omega_{1}, \Omega_{3}\right)-\eta\left(\Omega_{3}\right) \sigma\left(\Omega_{1}, \Omega_{2}\right) .
$$

Suppose that, $N$ is parallel with respect to LCC $\widetilde{\nabla}$. Then, from (3.2) we have

$$
\left(\overline{\widetilde{\nabla}}_{\Omega_{1}} \sigma\right)\left(\Omega_{2}, \Omega_{3}\right)=-\eta\left(\Omega_{2}\right) \sigma\left(\Omega_{1}, \Omega_{3}\right)-\eta\left(\Omega_{3}\right) \sigma\left(\Omega_{1}, \Omega_{2}\right) .
$$

Thus $N$ is not parallel with respect to SSMC $\overline{\widetilde{\nabla}}$.

We also state following result.

Corollary 3.4. Let $M$ be an $N K(Q E)_{m}$ manifold with respect to $S S M C \overline{\widetilde{\nabla}}, N$ be a submanifold of $M$ and $\xi$ be a parallel vector field with respect to $S S M C \overline{\widetilde{\nabla}}$. If $N$ is parallel with respect to $S S M C \overline{\widetilde{\nabla}}$ then it is not parallel with respect to LCC $\widetilde{\nabla}$. 
The Codazzi equation for $N$ is given by

$$
\widetilde{\operatorname{Rim}}\left(\Omega_{1}, \Omega_{2}, \Omega_{3}, \Omega_{4}\right)=\operatorname{Rim}\left(\Omega_{1}, \Omega_{2}, \Omega_{3}, \Omega_{4}\right)+g\left(\sigma\left(\Omega_{1}, \Omega_{3}\right), \sigma\left(\Omega_{2}, \Omega_{4}\right)\right)-g\left(\sigma\left(\Omega_{2}, \Omega_{3}\right), \sigma\left(\Omega_{1}, \Omega_{4}\right)\right)
$$

for all $\Omega_{1}, \Omega_{2}, \Omega_{3}, \Omega_{4} \in \Gamma(T N)$, where $\widetilde{\operatorname{Rim}}$ is the Riemannian curvature tensor of $M$ and Rim is the Riemannian curvature tensor of $N$ [1]. Let $M$ be an $N K(Q E)_{m}$ manifold with respect to SSMC $\overline{\widetilde{\nabla}}, \xi$ be a parallel vector field with respect to SSMC $\overline{\widetilde{\nabla}}$ and $N$ be a submanifold of $M$. From (2.4) and (3.2), we get

$$
\begin{aligned}
\overline{\widetilde{R i m}}\left(\Omega_{1}, \Omega_{2}, \Omega_{3}, \Omega_{4}\right) & =\operatorname{Rim}\left(\Omega_{1}, \Omega_{2}, \Omega_{3}, \Omega_{4}\right)+g\left(\sigma\left(\Omega_{1}, \Omega_{3}\right) \sigma\left(\Omega_{2}, \Omega_{4}\right)\right)-g\left(\sigma\left(\Omega_{2}, \Omega_{3}\right) \sigma\left(\Omega_{1}, \Omega_{4}\right)\right. \\
& +g\left(\Omega_{2}, \Omega_{3}\right) g\left(\Omega_{1}, \Omega_{4}\right)-g\left(\Omega_{1}, \Omega_{3}\right) g\left(\Omega_{2}, \Omega_{4}\right) .
\end{aligned}
$$

Thus , by using (2.5) we obtain

$$
\operatorname{Rim}\left(\Omega_{1}, \Omega_{2}, \Omega_{3}, \Omega_{4}\right)=\frac{a+b}{m-1}\left[g\left(\Omega_{2}, \Omega_{3}\right) g\left(\Omega_{1}, \Omega_{4}\right)+g\left(\Omega_{1}, \Omega_{3}\right) g\left(\Omega_{2}, \Omega_{4}\right)\right]-g\left(\sigma\left(\Omega_{1}, \Omega_{3}\right), \sigma\left(\Omega_{2}, \Omega_{4}\right)\right)+g\left(\sigma\left(\Omega_{2}, \Omega_{3}\right), \sigma\left(\Omega_{1}, \Omega_{4}\right)\right]
$$

Finally, we state the following theorem.

Theorem 3.5. Let $M$ be an $N K(Q E)_{m}$ manifold with respect to $S S M C \overline{\widetilde{\nabla}}, N$ be a submanifold of $M$ and $\xi$ be a parallel vector field with respect to $S S M C \overline{\widetilde{\nabla}}$. If $N$ is totally geodesic, then $N$ is an $N K(Q E)_{m}$ manifold with $k=\frac{a+b}{m-1}$.

On the other hand if $N$ is totally umbilical, i.e. $\sigma\left(\Omega_{1}, \Omega_{2}\right)=H g\left(\Omega_{1}, \Omega_{2}\right)$, then we get

$$
\operatorname{Rim}\left(\Omega_{1}, \Omega_{2}, \Omega_{3}, \Omega_{4}\right)=\left(\frac{a+b}{m-1}+g(H, H)\right)\left[g\left(\Omega_{2}, \Omega_{3}\right) g\left(\Omega_{1}, \Omega_{4}\right)+g\left(\Omega_{1}, \Omega_{3}\right) g\left(\Omega_{2}, \Omega_{4}\right)\right] .
$$

where $H$ is the mean curvature of $N$. Therefore we can state following theorem.

Theorem 3.6. Let $M$ be an $N K(Q E)_{m}$ manifold with respect to $S S M C \overline{\widetilde{\nabla}}, N$ be a submanifold of $M$ and $\xi$ be a parallel vector field with respect to SSMC $\overline{\widetilde{\nabla}}$. If $N$ is totally umbilical, then $N$ is a generalized real space form.

Example 3.7. Let $M$ be a $(2 m+1)$-dimensional smooth manifold. $(\phi, \xi, \eta)$ is called an almost para-contact structure on $M$ such that

$$
\phi^{2} \Omega=\Omega-\eta(\Omega) \xi, \phi(\xi)=0, \quad \eta \circ \phi=0, \quad \eta(\xi)=1
$$

where $\phi$ is a $(1,1)$ tensor field, $\xi$ is a vector field, $\eta$ is a $1-$ form, and $\Omega$ is an arbitrary vector field on M [18]. M is called a para-Kenmotsu $(P K)$ manifold if we have

$$
\left(\widetilde{\nabla}_{\Omega_{1}} \phi\right) \Omega_{2}=-g\left(\phi \Omega_{1}, \Omega_{2}\right) \xi+\eta\left(\Omega_{2}\right) \phi \Omega_{1}
$$

for all $\Omega_{1}, \Omega_{2} \in \Gamma(T M)$ [14]. Thus on $M$, we have

$$
\widetilde{\nabla}_{\Omega_{1}} \xi=-\phi^{2} \Omega_{1}
$$

for all $\Omega_{1} \in \Gamma(T M)$.

Let $\overline{\widetilde{\nabla}}$ be a SSMC defined in (2.1) on M. Thus, we get $\overline{\widetilde{\nabla}}_{\Omega_{1}} \xi=0$, i.e $\xi$ is parallel with respect to SSMC $\overline{\widetilde{\nabla}}$.

The $\phi$-sectional curvature of PK-manifold is defined as the sectional curvature of plane section spanned by $\Omega_{1}$ and $\phi \Omega_{1}$, for unit vector field $\Omega_{1}$. If $M$ has constant $\phi$-sectional curvature c then we have

$$
\begin{aligned}
\widetilde{\operatorname{Rim}}\left(\Omega_{1}, \Omega_{2}, \Omega_{3}, \Omega_{4}\right) & =\left(\frac{c-3}{4}\right)\left[g\left(\Omega_{2}, \Omega_{3}\right) g\left(\Omega_{1}, \Omega_{4}\right)-g\left(\Omega_{1}, \Omega_{3}\right) g\left(\Omega_{2}, \Omega_{4}\right)\right] \\
& +\left(\frac{c+1}{4}\right)\left[g\left(\Omega_{1}, \phi \Omega_{3}\right) g\left(\phi \Omega_{2}, \Omega_{4}\right)-g\left(\Omega_{1}, \phi \Omega_{3}\right) g\left(\phi \Omega_{2}, \Omega_{4}\right)+2 g\left(\Omega_{1}, \phi \Omega_{2}\right) g\left(\phi \Omega_{3}, \Omega_{4}\right),\right. \\
& \left.+\eta\left(\Omega_{1}\right) \eta\left(\Omega_{3}\right) g\left(\Omega_{2}, \Omega_{4}\right)-\eta\left(\Omega_{2}\right) \eta\left(\Omega_{3}\right) g\left(\Omega_{1}, \Omega_{4}\right)+g\left(\Omega_{1}, \Omega_{3}\right) \eta\left(\Omega_{2}\right) \eta\left(\Omega_{4}\right)-g\left(\Omega_{2}, \Omega_{3}\right) \eta\left(\Omega_{1}\right) \eta\left(\Omega_{4}\right)\right] .
\end{aligned}
$$

A PK-manifold $M$ with above curvature relation is called a PK-space form. For details see [13]. The Ricci curvature of a PK-space forms is given by

$$
\widetilde{\operatorname{Ric}}\left(\Omega_{1}, \Omega_{2}\right)=\left(\frac{(m+1)(c+1)}{4}-(m-1)\right) g\left(\Omega_{1}, \Omega_{2}\right)-\frac{(m+1)(c+1)}{4} \eta\left(\Omega_{1}\right) \eta\left(\Omega_{2}\right) .
$$

This shows $M$ is a quasi-Einstein manifold with $a=\frac{(m+1)(c+1)}{4}-(m-1), b=\frac{(m+1)(c+1)}{4}$. On a PK-manifold we have

$$
\left(\tilde{\nabla}_{\Omega_{1}} \eta\right) \Omega_{2}=g\left(\Omega_{1}, \Omega_{2}\right)-\eta\left(\Omega_{1}\right) \eta\left(\Omega_{2}\right),
$$

thus we obtain

$$
\omega\left(\Omega_{1}, \Omega_{2}\right)=\frac{3}{2} g\left(\Omega_{1}, \Omega_{2}\right)-2 \eta\left(\Omega_{1}\right) \eta\left(\Omega_{2}\right) .
$$

By using (2.2), the curvature of a PK-manifold admitting SSMC given in (2.1) is

$$
\begin{aligned}
\widetilde{\widetilde{R i m}}\left(\Omega_{1}, \Omega_{2}, \Omega_{3}, \Omega_{4}\right) & =\widetilde{\operatorname{Rim}}\left(\Omega_{1}, \Omega_{2}, \Omega_{3}, \Omega_{4}\right)-3\left(g\left(\Omega_{2}, \Omega_{3}\right) g\left(\Omega_{1}, \Omega_{4}\right)-g\left(\Omega_{1}, \Omega_{3}\right) g\left(\Omega_{2}, \Omega_{4}\right)\right. \\
& \left.+\eta\left(\Omega_{1}\right) \eta\left(\Omega_{3}\right) g\left(\Omega_{2}, \Omega_{4}\right)-\eta\left(\Omega_{2}\right) \eta\left(\Omega_{3}\right) g\left(\Omega_{1}, \Omega_{4}\right)+\eta\left(\Omega_{2}\right) \eta\left(\Omega_{4}\right) g\left(\Omega_{1}, \Omega_{3}\right)-\eta\left(\Omega_{1}\right) \eta\left(\Omega_{4}\right) g\left(\Omega_{2}, \Omega_{3}\right)\right) .
\end{aligned}
$$


Also, from (3.7), on a PK-space form we get

$$
\begin{aligned}
\widetilde{\widetilde{\operatorname{Rim}}}\left(\Omega_{1}, \Omega_{2}, \Omega_{3}, \Omega_{4}\right) & =\left(\frac{c-15}{4}\right)\left(g\left(\Omega_{2}, \Omega_{3}\right) g\left(\Omega_{1}, \Omega_{4}\right)-g\left(\Omega_{1}, \Omega_{3}\right) g\left(\Omega_{2}, \Omega_{4}\right)\right. \\
& +\left(\frac{c-11}{4}\right) \eta\left(\Omega_{1}\right) \eta\left(\Omega_{3}\right) g\left(\Omega_{2}, \Omega_{4}\right)-\eta\left(\Omega_{2}\right) \eta\left(\Omega_{3}\right) g\left(\Omega_{1}, \Omega_{4}\right)+\left(\eta\left(\Omega_{2}\right) \eta\left(\Omega_{4}\right) g\left(\Omega_{1}, \Omega_{3}\right)\right. \\
& \left.+\eta\left(\Omega_{1}\right) \eta\left(\Omega_{4}\right) g\left(\Omega_{2}, \Omega_{3}\right)\right) \\
& +\left(\frac{c+1}{4}\right)\left[g\left(\Omega_{1}, \phi \Omega_{3}\right) g\left(\phi \Omega_{2}, \Omega_{4}\right)-g\left(\Omega_{1}, \phi \Omega_{3}\right) g\left(\phi \Omega_{2}, \Omega_{4}\right)+2 g\left(\Omega_{1}, \phi \Omega_{2}\right) g\left(\phi \Omega_{3}, \Omega_{4}\right)\right]
\end{aligned}
$$

A generalized para-Sasakian space form (GPSSF) is an almost para-contact metric manifold $(M, \phi, \xi, \eta, g)$ with the following curvature relation;

$$
\begin{aligned}
\widetilde{\operatorname{Rim}}\left(\Omega_{1}, \Omega_{2}, \Omega_{3}, \Omega_{4}\right) & =F_{1}\left[g\left(\Omega_{2}, \Omega_{3}\right) g\left(\Omega_{1}, \Omega_{4}\right)-g\left(\Omega_{1}, \Omega_{3}\right) g\left(\Omega_{2}, \Omega_{4}\right)\right] \\
& +F_{2}\left(-g\left(\Omega_{1}, \phi \Omega_{3}\right) g\left(\phi \Omega_{2}, \Omega_{4}\right)+g\left(\Omega_{1}, \phi \Omega_{3}\right) g\left(\phi \Omega_{2}, \Omega_{4}\right)-2 g\left(\Omega_{1}, \phi \Omega_{2}\right) g\left(\phi \Omega_{3}, \Omega_{4}\right)\right) \\
& \times F_{3}\left(\eta\left(\Omega_{1}\right) \eta\left(\Omega_{3}\right) g\left(\Omega_{2}, \Omega_{4}\right)-\eta\left(\Omega_{2}\right) \eta\left(\Omega_{3}\right) g\left(\Omega_{1}, \Omega_{4}\right)+g\left(\Omega_{1}, \Omega_{3}\right) \eta\left(\Omega_{2}\right) \eta\left(\Omega_{4}\right)-g\left(\Omega_{2}, \Omega_{3}\right) \eta\left(\Omega_{1}\right) \eta\left(\Omega_{4}\right)\right)
\end{aligned}
$$

for all $\Omega_{1}, \Omega_{2}, \Omega_{3}, \Omega_{4}$ vector fields.

Corollary 3.8. A PK-space form with respect to SSMC $\overline{\widetilde{\nabla}}$ is a GPSSF with $F_{1}=\frac{c-15}{4}, F_{2}=-\frac{c-11}{4}$ and $F_{3}=\frac{c+1}{4}$.

Let take an orthonormal basis of $M$ by $E_{1}, E_{2}, \ldots E_{n}, E_{m+1}=\phi E_{1}, \ldots, E_{2 m}=\phi E_{m}, \xi$. By choosing $\Omega_{2}=\Omega_{3}=E_{i}$ and taking sum over $i$ such that $1 \leq i \leq 2 m$ in (3.11) then, we obtain

$$
\widetilde{\widetilde{R i c}}\left(\Omega_{1}, \Omega_{2}\right)=\left(\frac{m(c-15)-2}{2}\right) g\left(\Omega_{1}, \Omega_{2}\right)+\frac{c-11}{4}(1-2 m) \eta\left(\Omega_{1}\right) \eta\left(\Omega_{4}\right) \text {. }
$$

Thus, $M$ is a quasi-Einstein manifold. So, we state;

Corollary 3.9. A PK-space form with respect to SSMC $\overline{\widetilde{\nabla}}$ is a quasi-Einstein manifold.

This is compatible with Theorem 2.2.

Let $N$ be a submanifold of $P K$-space form $M$ with respect to $\overline{\widetilde{\nabla}}$. Then, we have

$$
\begin{aligned}
\operatorname{Rim}\left(\Omega_{1}, \Omega_{2}, \Omega_{3}, \Omega_{4}\right) & =\widetilde{\operatorname{Rim}}\left(\Omega_{1}, \Omega_{2}, \Omega_{3}, \Omega_{4}\right)-g\left(\sigma\left(\Omega_{1}, \Omega_{3}\right) \sigma\left(\Omega_{2}, \Omega_{4}\right)\right)+g\left(\sigma\left(\Omega_{2}, \Omega_{3}\right) \sigma\left(\Omega_{1}, \Omega_{4}\right)\right. \\
& -g\left(\Omega_{2}, \Omega_{3}\right) g\left(\Omega_{1}, \Omega_{4}\right)+g\left(\Omega_{1}, \Omega_{3}\right) g\left(\Omega_{2}, \Omega_{4}\right)
\end{aligned}
$$

and from (3.11) we get

$$
\begin{aligned}
\operatorname{Rim}\left(\Omega_{1}, \Omega_{2}, \Omega_{3}, \Omega_{4}\right) & =\left(\frac{c-19}{4}\right)\left(g\left(\Omega_{2}, \Omega_{3}\right) g\left(\Omega_{1}, \Omega_{4}\right)-g\left(\Omega_{1}, \Omega_{3}\right) g\left(\Omega_{2}, \Omega_{4}\right)\right. \\
& +\left(\frac{c-11}{4}\right)\left(\eta\left(\Omega_{1}\right) \eta\left(\Omega_{3}\right) g\left(\Omega_{2}, \Omega_{4}\right)-\eta\left(\Omega_{2}\right) \eta\left(\Omega_{3}\right) g\left(\Omega_{1}, \Omega_{4}\right)\right. \\
& \left.+\eta\left(\Omega_{2}\right) \eta\left(\Omega_{4}\right) g\left(\Omega_{1}, \Omega_{3}\right)-\eta\left(\Omega_{1}\right) \eta\left(\Omega_{4}\right) g\left(\Omega_{2}, \Omega_{3}\right)\right) \\
& +\left(\frac{c+1}{4}\right)\left(g\left(\Omega_{1}, \phi \Omega_{3}\right) g\left(\phi \Omega_{2}, \Omega_{4}\right)-g\left(\Omega_{1}, \phi \Omega_{3}\right) g\left(\phi \Omega_{2}, \Omega_{4}\right)+2 g\left(\Omega_{1}, \phi \Omega_{2}\right) g\left(\phi \Omega_{3}, \Omega_{4}\right)\right) \\
& -g\left(\sigma\left(\Omega_{1}, \Omega_{3}\right) \sigma\left(\Omega_{2}, \Omega_{4}\right)\right)+g\left(\sigma\left(\Omega_{2}, \Omega_{3}\right) \sigma\left(\Omega_{1}, \Omega_{4}\right)\right.
\end{aligned}
$$

for all $\Omega_{1}, \Omega_{2}, \Omega_{3}, \Omega_{4} \in \Gamma(T N)$.

Suppose that $\xi$ is normal to $N$ and $N$ is an anti-invariant submanifold i.e. $\phi \Omega_{1} \in \Gamma\left(T N^{\perp}\right)$, for $\Omega_{1} \in \Gamma(T N)$. Then, we get

$$
\operatorname{Rim}\left(\Omega_{1}, \Omega_{2}, \Omega_{3}, \Omega_{4}\right)=\left(\frac{c-19}{4}\right)\left(g\left(\Omega_{2}, \Omega_{3}\right) g\left(\Omega_{1}, \Omega_{4}\right)-g\left(\Omega_{1}, \Omega_{3}\right) g\left(\Omega_{2}, \Omega_{4}\right)+g\left(\sigma\left(\Omega_{1}, \Omega_{3}\right) \sigma\left(\Omega_{2}, \Omega_{4}\right)\right)-g\left(\sigma\left(\Omega_{2}, \Omega_{3}\right) \sigma\left(\Omega_{1}, \Omega_{4}\right) .\right.\right.
$$

Thus, we state following results.

Corollary 3.10. Let $M$ be a PK-space form with respect to SSMC $\overline{\widetilde{\nabla}}$ and $N$ be an anti-invariant submanifold of $M$ with $\xi$ is normal to $N$. If $N$ is totally geodesic, then $N$ is $N(k)$-manifold.

Corollary 3.11. Let $M$ be a PK-space form with respect to SSMC $\overline{\widetilde{\nabla}}$ and $N$ be an anti-invariant submanifold of $M$ with $\xi$ is normal to $N$. If $N$ is totally umbilical, then $N$ is a reel space form.

Corollary 3.12. Let $M$ be a PK-space form with respect to SSMC $\overline{\widetilde{\nabla}}$ and $N$ be an anti-invariant submanifold of $M$ with $\xi$ is normal to $N$. If $N$ is totally geodesic. Then $N$ is an Einstein manifold.

Let $M$ be a PK-space form with respect to SSMC $\overline{\widetilde{\nabla}}$ and $N$ be a submanifold of $M$. If $\xi$ is tangent to submanifold $N$, then Lemma 3.1 is verified. Also, for the same submanifold the Theorem 3.3 is verified. 


\section{References}

[1] K. Yano, M. Kon, Structures on Manifolds, Series in Pure Mathematics, World Scientific, 3, 1984.

[2] C. Özgür , M. M. Tripathi, On the concircular curvature tensor of an N( $\kappa)$-quasi Einstein manifold, Math. Pannon., 18(1), (2007), 95-100.

[3] C. Özgür, $N(\kappa)$-quasi Einstein manifolds satisfying certain conditions, Chaos Solitons Fractals, 38(5) (2008), $1373-1377$.

[4] A. Yıldız, U.C. De, A. Çetinkaya, On some classes of $N(\kappa)$-quasi Einstein manifolds, Proc. Natl. Acad. Sci. India A, 83(3) (2013), $239-245$.

[5] M.C. Chaki, On quasi Einstein manifolds, Publ. Math. Debr., 57 (2000), 297-306.

[6] S.K. Chaubey, Existence of $N(\kappa)$-quasi Einstein manifolds, Facta universitatis Nis. Ser. Math.Inform., 32(3) (2017), 369-385.

[7] U.C. De, G.C.Ghosh, On quasi Einstein manifolds, Period. Math. Hung., 48 (2004), 223-231.

[8] U. C. De, S. Shenawy, Generalized quasi-Einstein GRW space-times, Int. J. Geom. Methods Mod. Phys., 16(08) (2019), 1950124.

[9] G.C. Ghosh, U.C. De, T.Q. Binh, Certain curvature restrictions on a quasi Einstein manifolds, Publ. Math. Debr. 69 (2006), $209-217$.

[10] A.T. Kotamkar, A. Tarini, T. Brajendra, Certain curvature conditions catisfied by $N(\kappa)$-quasi Einstein manifolds, Int. J. Innov. Res. Adv. Eng. G. , 1(9) (2015), 1-9.

[11] C. Murathan, C. Özgür, Riemannian manifolds with a semi-symmetric metric connection satisfying some semi-symmetry conditions, Proc. Est. Acad. Sci., 57(4) (2008), 210-216.

[12] H.G. Nagaraja, K. Venu, On Ricci solitons in N( )-quasi Einstein manifolds, NTMSCI, 5(3) (2017), 46-52.

[13] G. Pitiş, Geometry of Kenmotsu Manifolds, Editura Universitatii Transilvania, 2007.

[14] B.B. Sinha, K. L. Sai Prasad, A class of almost para contact metric manifolds, Bull. Cal. Math. Soc., 87 (1995), $307-312$.

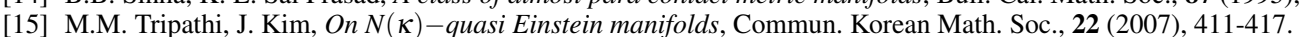

[16] A. Taleshian, A. A. Hosseinzadeh, Investigation of some conditions on N( $\kappa)$-quasi Einstein manifolds, Bull. Malaysian Math. Sci. Soc, 34(3) (2011),

[17] K. Yano, On semi-symmetric connection, Revue Roumaine Math. Pures Appl., 15 (1970), 1570-1586.

[18] S. Zamkovoy, Canonical connections on paracontact manifolds, Ann. Global Anal. Geom., 36(1) (2008), 37-60. 